\title{
Calibration of the fundamental diagram based on loop and probe data
}

\author{
Aurélien Clairais \\ University of Lyon, F-69000, Lyon, France \\ IFSTTAR, LICIT, F-69500, Bron \\ ENTPE, LICIT, F-69518, Vaulx en Velin \\ phone: +33 (0) 472142375 , \\ aurelien.clairais@ifsttar.fr \\ Aurélien Duret (corresponding author) \\ University of Lyon, F-69000, Lyon, France \\ IFSTTAR, LICIT, F-69500, Bron \\ ENTPE, LICIT, F-69518, Vaulx en Velin \\ phone: +33 (0) 472142331 , \\ aurelien.duret@ifsttar.fr \\ Nour-Eddin El Faouzi \\ Université de Lyon, F-69000, Lyon, France \\ IFSTTAR, LICIT, F-69500, Bron \\ ENTPE, LICIT, F-69518, Vaulx en Velin \\ phone: +33 (0) 472142543 , \\ nour-eddin.elfaouzi@ifsttar.fr \\ Paper submitted to TRB Annual Meeting 2016 \\ July 31, 2015 \\ 3026 words +8 figure $(s)+3$ table $(s) \Rightarrow 5776$ 'words' \\ Notes: 1) Figures and Tables each count as 250 words. 2) Maximum of 7500 \\ 'words'.
}


1 ABSTRACT

The fundamental diagram is a key component of traffic flow. It describes equilibrium traffic states and their propagation on a traffic network. The knowledge of its parameters is of paramount importance to understand traffic properties and its characteristics. It is also critical to calibrate the elements of dynamic traffic flow simulation models and reproduce traffic states on road networks. The paper is concerned with developing a method for estimating fundamental diagram parameters that combines loop data and probe data.

Loop data are considered as boundary conditions of the problem. Travel times between (any points located between) the loop can be estimated based on the fundamentals of the kinematic wave theory. The optimal fundamental diagram parameters are computed so that the discrepancy between estimated travel times and actual travel times from probes is minimal.

The method is validated on simulated error-free data. The results demonstrate the accuracy of the method when applied on an error-free dataset. The method is then implemented using realistic data, i.e. aggregated and noised beforehand. It demonstrates its robustness and the results are encouraging for developing a algorithm that calibrates online and automatically fundamental diagram parameters. 


\section{INTRODUCTION}

\section{Calibration of the fundamental diagram: state of the art}

The Fundamental Diagram (FD) relates traffic flow and density. Under time-stationary and spacehomogeneous conditions, it describes equilibrium traffic states and their propagation on a traffic network. The knowledge of its functional form and parameters is of paramount importance to understand traffic properties. It is also a key element of dynamic traffic flow simulation models that aim to reproduce traffic conditions on road networks.

The early studies (1930-1970) were based on the analysis of flow and speed observed at fixed locations of the network. Many functional forms have been proposed to describe the speedflow relationship and interested reader may refer to $(I)$ for a comprehensive overview. Then speedflow and flow-density relationships have been estimated based on data collected from loop detectors, commonly aggregated over space (multilanes) and time (2, 3, 4). However, three drawbacks can be pointed out when loop data is used (5): (i) aggregated data presents mixed traffic states that cannot be considered as equilibrium, (ii) Steady state can be difficult to capture and (iii) the state propagations cannot be capture at fixed location.

Alternatively, (6) proposed a method to identify steady state periods in order not to mix different traffic states measured by loops. The results presented by the author demonstrate the capability of the method to capture traffic steady-states. However, the method requires manual intervention to adjust the steady state period and can hardly be automated.

More recently, (7) have proposed to estimate FD parameters by capturing wave propagation through successive loops. This method has proved its ability to capture traffic dynamics and estimate the FD, in particular its congested branch. However, it requires data to be collected on successive loops without any entry or exit in between, which limits its practical use.

During the last decade, trajectory dataset (8) have been widely used to analyze microscopic speed-spacing relationship $(9,10,11,12)$, which is closely related to the macroscopic flow-density relationship. (13) have proposed to derive macroscopic FD parameters from individual car-following parameters. Despite technological advances, trajectory data collection is still expensive to collect and requires time-consuming data post processing.

However, intelligent Transportation Systems have brought a new generation of Lagrangian traffic data: probe data. Probes provide Lagrangian observations such as individual position, speed and travel times. They are highly complementary to loop sensors, which provide Eulerian observations relative to the stream (flow-density-speed). During the last few years, the literature provided proposals using probe and loop data, mainly for traffic state estimation or travel time estimation $(14,15,16,17)$. However, no method has yet been proposed to calibrate FD parameters combining probe and loop data. This is the purpose of this paper, which objectives is to pave the way to rethink and refine existing estimation methods and to propose a new method for estimating FD.

\section{Traffic flow model}

The LWR model introduced by (18) and (19) describes the evolution of traffic density $k$ in time and space with a conservative form:

$$
\frac{\partial k}{\partial t}+\frac{\partial Q(k)}{\partial x}=0
$$

$Q(k)$ is the fundamental diagram, which is assumed to be triangular and time-space invariant in this paper. Its parameters are: $u$ the free-flow speed, $w$ the wave speed and $k_{x}$ the jam density. 
The LWR model is an initial-value problem. Given initial/boundary conditions, the model propagates traffic states in time and space. It is possible to solve the problem by using the method of characteristics. A characteristic is a curve that starts from a known initial/boundary condition and along which the quantity $k$ is invariant. A discontinuity in the initial/boundary conditions initiate shockwave traveling in time and space with a speed $c$, given by the Rankine-Hugoniot formula:

$$
c=\frac{q_{B}-q_{A}}{k_{B}-k_{A}}
$$

, where $A\left(q_{A}, k_{A}\right)$ and $B\left(q_{B}, k_{B}\right)$ are the traffic states around the discontinuity. Given a wellposed definition of initial/boundary conditions, it is possible to determine $k(x, t)$ everywhere in the solution domain by the method of characteristics (20).

\section{Objectives and content of the paper}

This paper aims to propose a method that estimates automatically FD parameters combining data from loops and probes. The method relies on the kinematic wave theory. In its current version, it requires two successive loop sensors without entry or exit in between and probe data collected on a section between the loops. In contrast to the previous method proposed by ( 7 ), it does not require any wave propagation from one loop to another.

The paper is organized as follows. The recipe of the calibration methodology is presented in the next section. Then the method is validated in two-steps. First, the accuracy of the method is verified applying on synthetic error-free dataset. Second, the method is applied on synthetic aggregated-noised dataset. It illustrates the sensitivity of the results to the data resolution: aggregation period of loop data, frequency of probe data and penetration rate of probe vehicles. It also demonstrates the possibility of operational implementation. Finally, a discussion on operational implementation and other applications is proposed.

\section{RECIPE FOR CALIBRATING THE FD}

Let us consider a homogeneous link with a length $\mathrm{L}$. The recipe of the method is the following. First, traffic counts given by two loop detectors are considered as the boundary conditions of the problem. Second, traffic states between loops are estimated based on the method of the characteristics, for a given set of FD parameters. Third, probe data are emulated and then compared to observed probe data. Finally, the optimal FD parameters minimize the difference between emulated and observed probe data.

\section{Boundary conditions}

Let $\Delta(t)$ and $\Sigma(t)$ be the upstream flow (demand) and the downstream flow (supply) measured by two consecutive loop detectors. Considering $\Delta$ and $\Sigma$ as the initial/boundary conditions of the problem, it is then possible to estimate the traffic states at any point in between. The solution of the problem consists in propagating traffic states by using the method of characteristic.

\section{Traffic states propagation}

Upstream and downstream flows can be approximated as step functions with respect to time, with: $\Delta(i . \delta t)=q_{\Delta}^{i}$ and $\Sigma(j . \delta t)=q_{\Sigma}^{j}$.

If $\Delta(t)$ presents a discontinuity at the time $t=i . \delta t$, then a characteristic $\tilde{u}^{i}$ emanates from the 
upstream boundary conditions. Similarly, if $\Sigma(t)$ presents a discontinuity at the time $t=j . \delta t$, a characteristic called $\tilde{w}^{j}$ emanates from the downstream boundary conditions.

When a congestion occurs, the method of characteristics can then be applied to estimate the trajectory of the front wave, which separates upstream free-flow states from the downstream congested states. In the case of stepwise boundary conditions, the resulting front wave trajectory is piece-wise linear. Let $P_{n}=\left\{T_{n}, X_{n}\right\}$ be the $n^{\text {th }}$ coordinates of the front wave trajectory. We propose to calculate the coordinates of the point $P_{n+1}$ by using the Rankine-Hugoniot formula as illustrated in Figure 1.

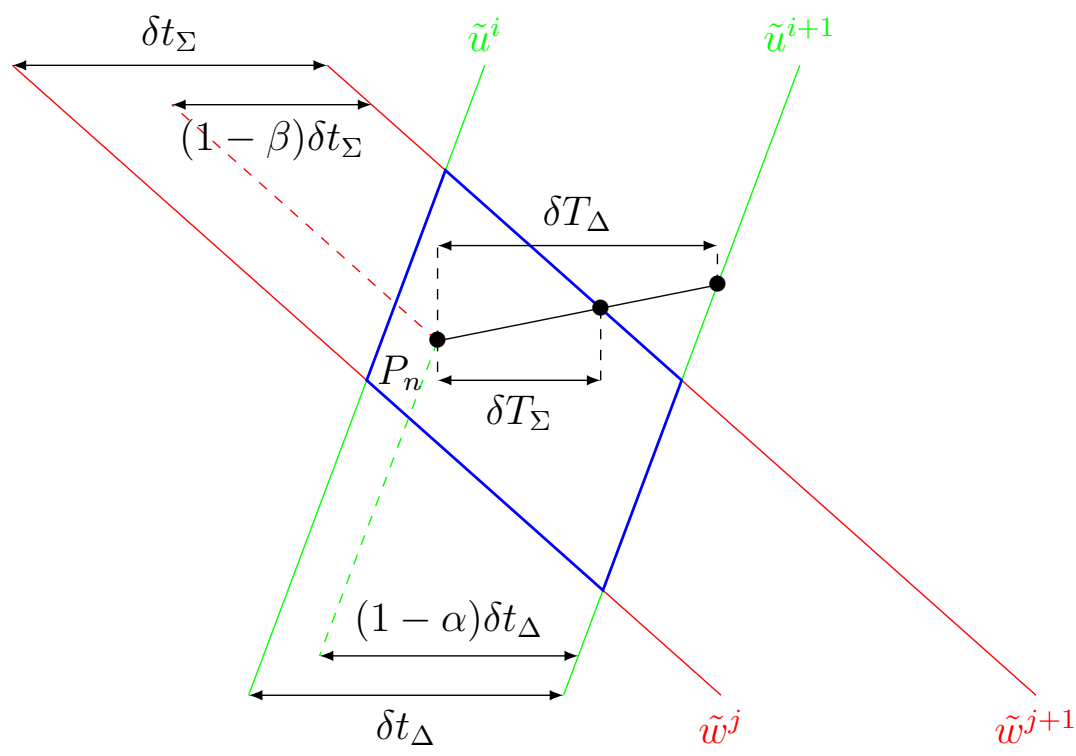

FIGURE 1 Research of the point $P_{n+1}$

9 10

Graphically, $P_{n}$ is located between the characteristics $\tilde{u}^{i}, \tilde{u}^{i+1}, \tilde{w}^{j}$ and $\tilde{w}^{j+1}$. A characteristic (black solid line in Figure 1) emanates from $P_{n}$ with the $W_{i, j}$.

One the one hand, the characteristic intersects $\tilde{u}^{i+1}$ which yields:

$$
\delta T_{\Delta}=\frac{(1-\alpha) \delta t_{\Delta}}{1-\frac{W_{i, j}}{u}}
$$

where

$$
\alpha=\frac{1}{\delta t_{\Delta}} \cdot\left(T_{n}-\frac{X_{n}}{u}-i . \delta t_{\Delta}\right)
$$

On the other hand, the characteristic intersect $\tilde{w}^{j+1}$, which yields:

$$
\delta T_{\Sigma}=\frac{(1-\beta) \delta t_{\Sigma}}{1+\frac{W_{i, j}}{w}}
$$

where

$$
\beta=\frac{1}{\delta t_{\Sigma}} .\left(T_{n}-\frac{L-X_{n}}{w}-j . \delta t_{\Sigma}\right)
$$




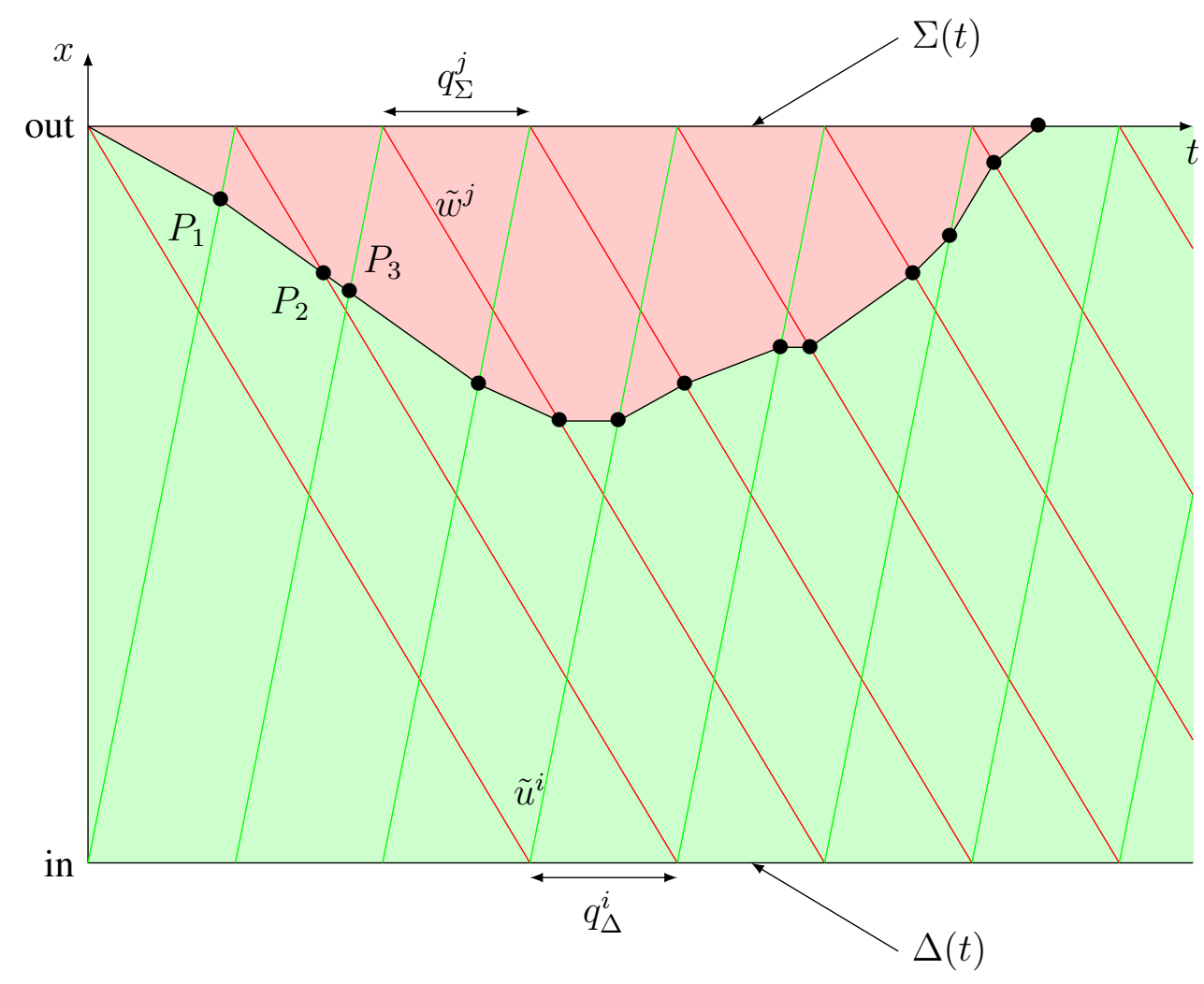

FIGURE 2 Front Wave Trajectory

The coordinates of the point $P_{n+1}$ follows:

$$
P_{n+1}=\left\{\begin{array}{l}
T_{n+1}=T_{n}+\min \left(\delta T_{\Delta}, \delta T_{\Sigma}\right) \\
X_{n+1}=X_{n}+W_{i, j} \cdot \min \left(\delta T_{\Delta}, \delta T_{\Sigma}\right)
\end{array}\right.
$$

The trajectory of the front wave can then been calculated by a recursive process, as illustrated in Figure 2.

The solution of the LWR model presented above finally provides traffic states of any intermediate location between loops. Upstream the front wave, traffic states emanate from the upstream boundary $\Delta(t)$ and travels with a speed $u$. Downstream the front wave, traffic states emanate from the downstream boundary $\Sigma(t)$ and travels with a speed $-w$.

\section{Simulation process of probe data}

We propose to simulate probe data based on the aforementioned solution. Commonly, probe data provides time-position of probe vehicles at fixed time intervals. It consists in successive timeposition, as illustrated in Figure 3 (red filled circles). $\left(t_{0}^{n}, x_{0}^{n}\right)$ and $\left(t_{1}^{n}, x_{1}^{n}\right)$ are the initial and the later position of the $n^{\text {th }}$ probe vehicle respectively. Considering the initial position of the probe vehicle $\left(t_{0}^{n}, x_{0}^{n}\right)$, the final position can easily be simulated from the results presented above. If the initial position $\left(t_{0}^{n}, x_{0}^{n}\right)$ is upstream the front wave, the vehicle is free-flowing and drives at the speed $u$. When it crosses the front wave, it turns into a congested state with a reduced speed and its motion is then driven by traffic conditions downstream. Then, its speed can change every time 


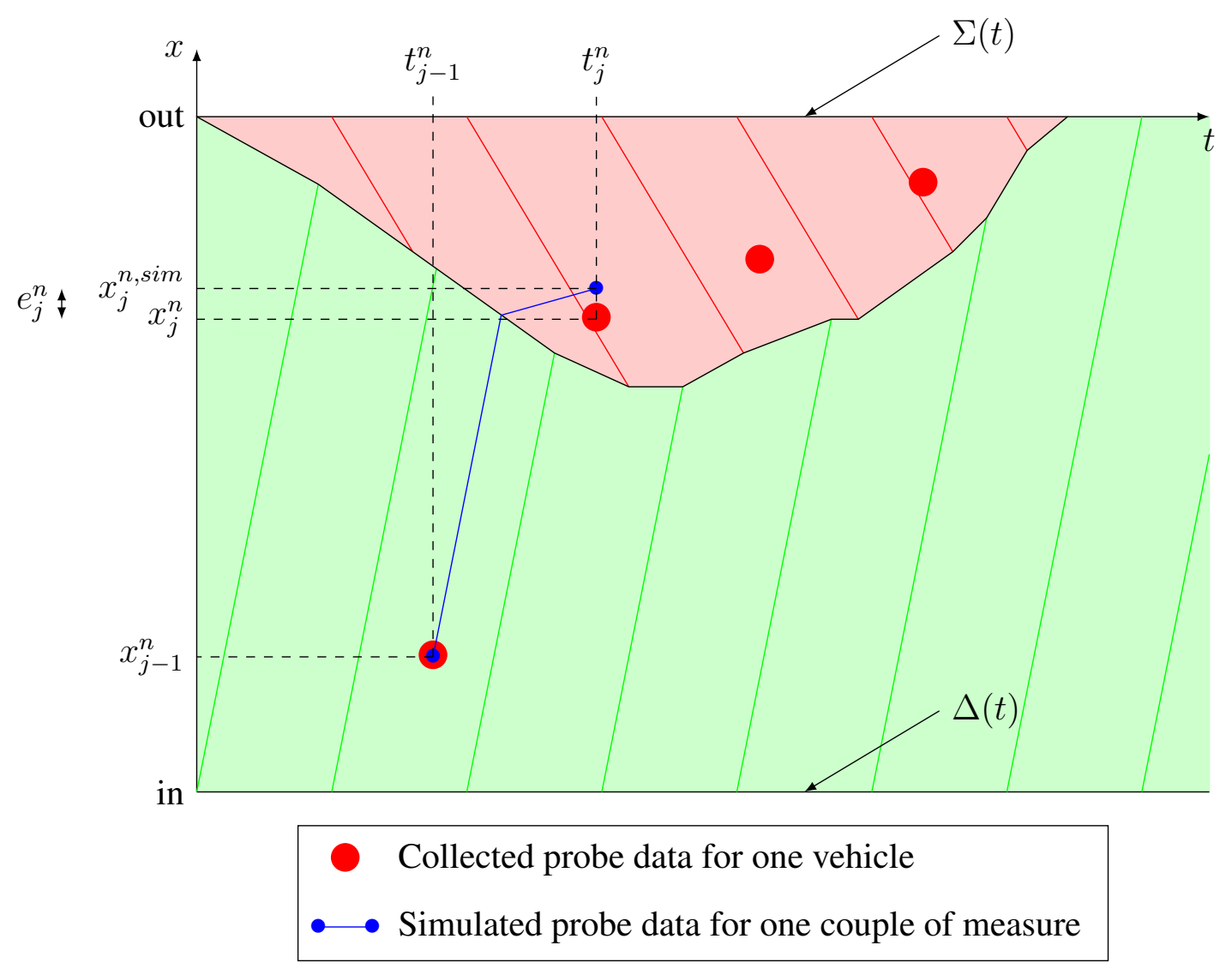

FIGURE 3 Calculation of $e_{j}^{n}\left(w, k_{x}\right)$ : discrepancy between collected and simulated probe data

a wave $\tilde{w}$ is crossed. Its final position $x_{1}^{n, s i m}$ at the later time $t_{1}^{n}$ can easily be derived, as illustrated in Figure 3 (blue circles).

\section{Calibration}

The calibration procedure aims to find the set of FD parameters that minimizes the discrepancy between simulated and observed probe data. The calibration of the free-flow speed is straightforward by using loop detectors only. Consequently the paper focuses on the calibration of congested parameters $w$ and $k_{x}$.

It should be noted that $x_{1}^{n, s i m}$ depends on the FD parameters: $w$ and $k_{x}$. Let $e_{j}^{n}\left(w, k_{x}\right)$ be the difference between emulated and observed position of the probe vehicle indexed $n$ at the time $t_{j}$.

$$
e_{j}^{n}\left(w, k_{x}\right)=x_{j}^{n}-x_{j}^{n, s i m}\left(w, k_{x}\right)
$$

We define the Goodness of Fit (GoF) as the root mean square error:

$$
R M S E\left(w, k_{x}\right)=\sqrt{\frac{1}{N} \sum_{n, j}\left(e_{j}^{n}\left(w, k_{x}\right)\right)^{2}}
$$

Here the RMSE assigns the same weight to each error. However, some errors are more valuable for capturing the traffic dynamics, in particular when probe vehicles move slowly and may cross 
one or several waves. Consequently, we propose to weight the errors according to probe speeds: heavy weights (denoted $\alpha$ ) are assigned to probe vehicles that present low observed mean speeds between the times $t_{0}$ and $t_{1}$. With normalized weights, the following GoF denoted $F\left(w, k_{x}\right)$ is considered:

$$
F\left(w, k_{x}\right)=\sqrt{\sum_{n, j} \alpha_{n} \cdot\left(e_{j}^{n}\left(w, k_{x}\right)\right)^{2}}
$$

Where :

$$
\left\{\begin{aligned}
\alpha_{n} & =\frac{u-\bar{v}^{n}}{\sum_{m}\left(u-\bar{v}^{n}\right)} \\
\bar{v}^{n} & =\frac{x_{j}^{n}-x_{j-1}^{n}}{t_{j}^{n}-t_{j-1}^{n}}
\end{aligned}\right.
$$

Finally, the optimal set of parameters $\left(w^{*}, k_{x}^{*}\right)$ minimize the function $F$.

\section{VALIDATION}

To validate the calibration procedure, we have performed two simulation studies. For the first simulation study, the calibration procedure is executed on an error-free dataset. For the second simulation study, the calibration procedure is performed on a set of realistic aggregated and noised data. The accuracy of the calibration procedure is tested regarding different observation models with varying aggregation period (loop), frequency (probe) and penetration rate (probe).

\section{Error-free dataset}

First, the calibration procedure is tested on synthetic loop and probe data that are error free. The calibration process is supposed to be exact and gives the FD parameter used by the simulation with $F^{*}=F\left(w^{*}, k_{x}^{*}\right)=0$.

Simulated data The idea is to let the mesoscopic-LWR model $(21,22)$ generate vehicle trajectories with mesoscopic coordinates, which are convenient to generate both loop and probe data (23). Loop and probe data are generated on a homogeneous link of $10000 \mathrm{~m}$ in length. For the simulation purpose, the FD is defined as triangular with the following parameters: $u=25 \mathrm{~m} / \mathrm{s}$, $w=5 \mathrm{~m} / \mathrm{s}$ and $k_{x}=0,14 \mathrm{veh} / \mathrm{m}$. The results of the mesoscopic LWR model return passing time $T(n, x)$ of every vehicle $n$ at discrete positions $x$. The simulation scenario is defined so that congestion is triggered at the exit of the section, propagates backward and then disappears before the end of the simulation. For the purpose of the test, the probe data have been generated with a frequency $f=1 / 60 s^{-1}$ and a penetration rate $\tau=10 \%$.

Calibration results The results of the calibration procedure has been applied to find the optimal set of parameters. A range a reasonable parameter values (according to their physical meaning) have been tested: $k_{x}=\{0.1, \ldots, 0.2\} v e h / m$ and $w=\{4, \ldots, 6\} \mathrm{m} / \mathrm{s}$. Figure 4(a) depicts the shape of the function $F\left(w, k_{x}\right)$. As expected, optimal parameters exactly correspond to the FD parameters used for the simulation scenario: $k_{x}^{*}=0.14 v \mathrm{eh} / \mathrm{m}, w^{*}=5 \mathrm{~m} / \mathrm{s}$ and $F^{*}=0$. It confirms the accuracy of the calibration procedure.Figure 4(b) illustrates the shape of function $F\left(w, k^{*}\right)$. It should be noted that the shape of the cost function $F$ is similar to those presented in (24), where the objective of the calibration process was to calibrate individual (vehicle) FD 
parameters based on vehicle trajectory dataset. The sensitivity of the calibration procedure is illustrated in Figures 4(b) and 4(c), which represents the function $F\left(w, k_{x}^{*}\right)$ and $F\left(w^{*}, k_{x}\right)$. They correspond to the vertical cross section of $F$ with $k_{x}=k^{*}$ and $w=w^{*}$ respectively.

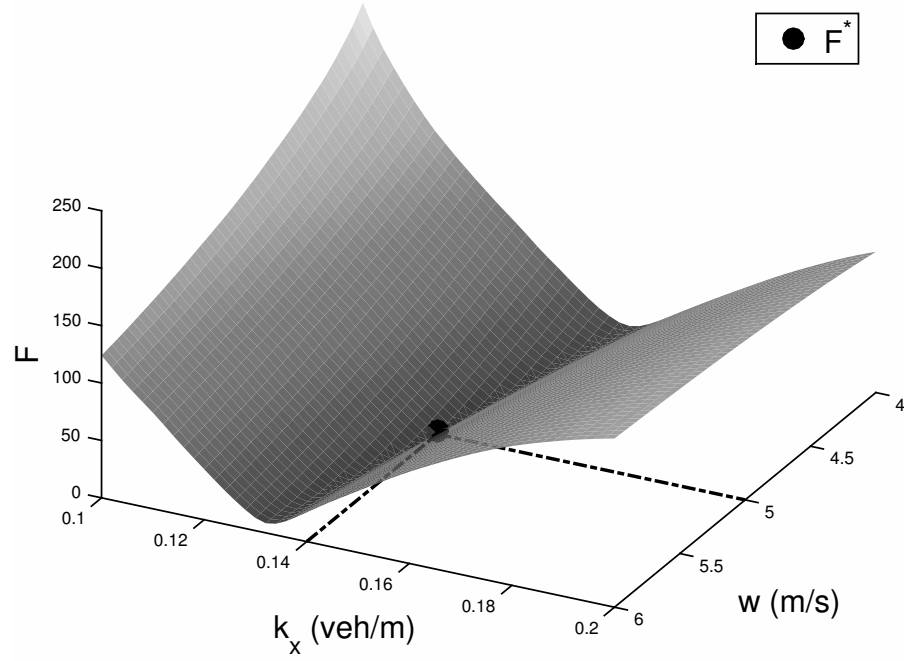

(a) $F\left(w, k_{x}\right)$

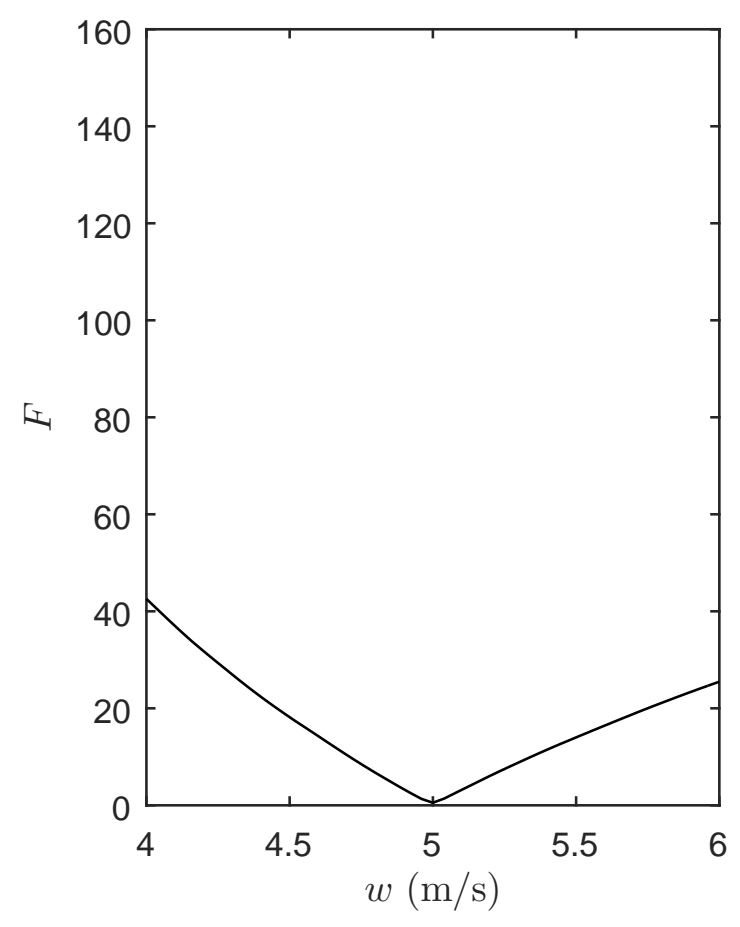

(b) $F\left(w, k_{x}^{*}\right)$

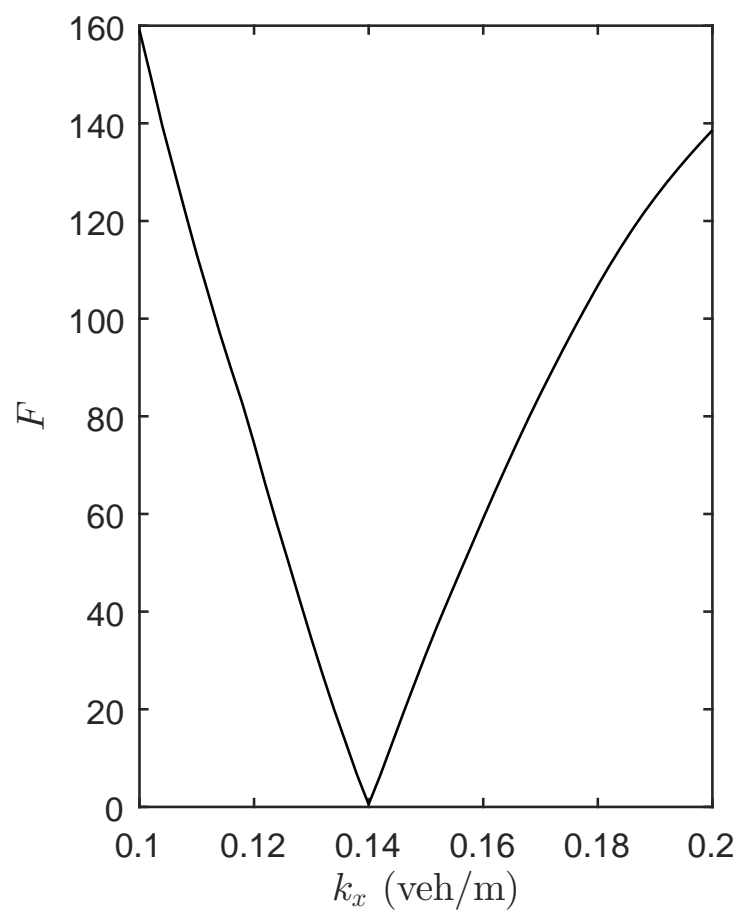

(c) $F\left(w^{*}, k_{x}\right)$

FIGURE 4 Results of the calibration for an error-free dataset

than $F\left(w^{*}, k_{x}\right)$. It demonstrates that the calibration process is more sensitive to errors on $k_{x}$. We conclude that the calibration of $k_{x}$ is more critical that $w$ in operational settings. It should be noted 
that these results can also be proved by an analytical sensitivity analysis.

\section{Realistic dataset}

The meso-LWR model is used to generate data on a realistic simulation scenario, and test the impact of data resolution on the calibration performance.

Efficiency and accuracy The extent to which a FD parameters can be accurately estimated is closely related to its ability to impact model predictions and thereby the value of the function $F$. As initially proposed by (25), here we analyse the shape of GoF regarding two indicators that estimate the efficiency and the accuracy of the calibration process.

The efficiency is quantified by the $F^{*}=F\left(w^{*}, k_{x}^{*}\right)$, which measures the ability of the model to fit the dataset.The efficiency of the calibration increases when $F^{*}$ decreases.

The accuracy is measured by the tightness of $F$ around optimal parameters, which represents the sensitivity of the calibration process to the error. The level of accuracy for parameter $p$ is denoted $A$ and is defined as the width of the function $F$ for $F=F^{*}+1$.

Simulated data The same link is considered. The simulation lasts three hours and the typical demand profiles are derived from a real dataset.

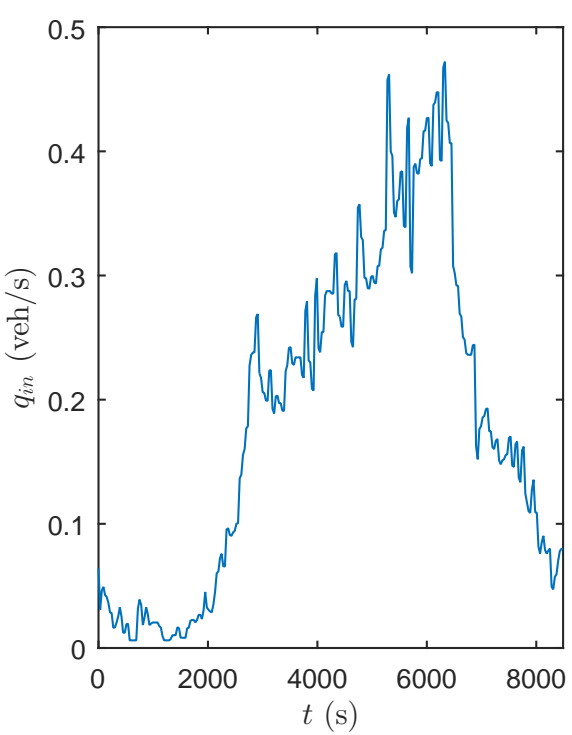

(a) Entry flow

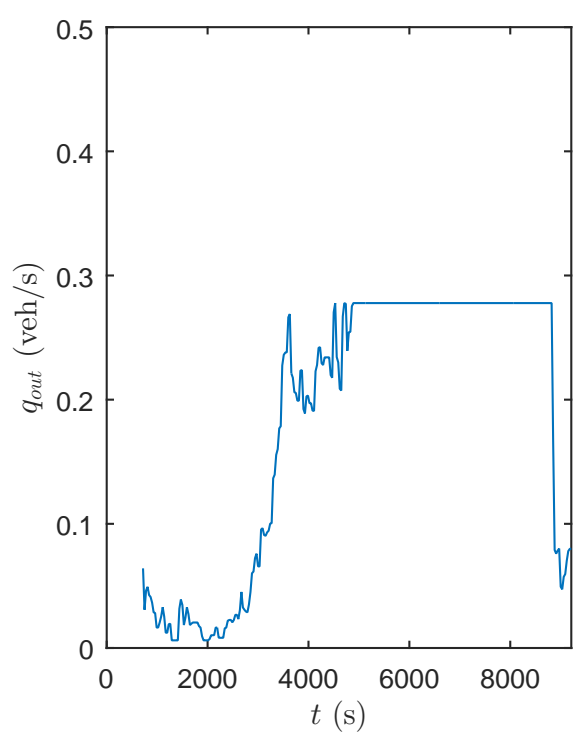

(b) Exit flow

\section{FIGURE 5 Flows at entry and exit of the link}

- Loop data are aggregated with $180 \mathrm{~s}$ interval. Loop data at entry and exit of the network are illustrated on Figure 5. Here loop data have been noised with unbiased errors as follows: each vehicle that crosses the loop detector has a probability of 0.01 to be undetected a probability of 0.01 to be detected twice.

- Probe data are measured with $f=1 / 60 s^{-1}$ and the penetration rate is set to $\tau=10 \%$.

Based on this data, three observation models have been considered to test the impact of data resolution and noise on the calibration results. 


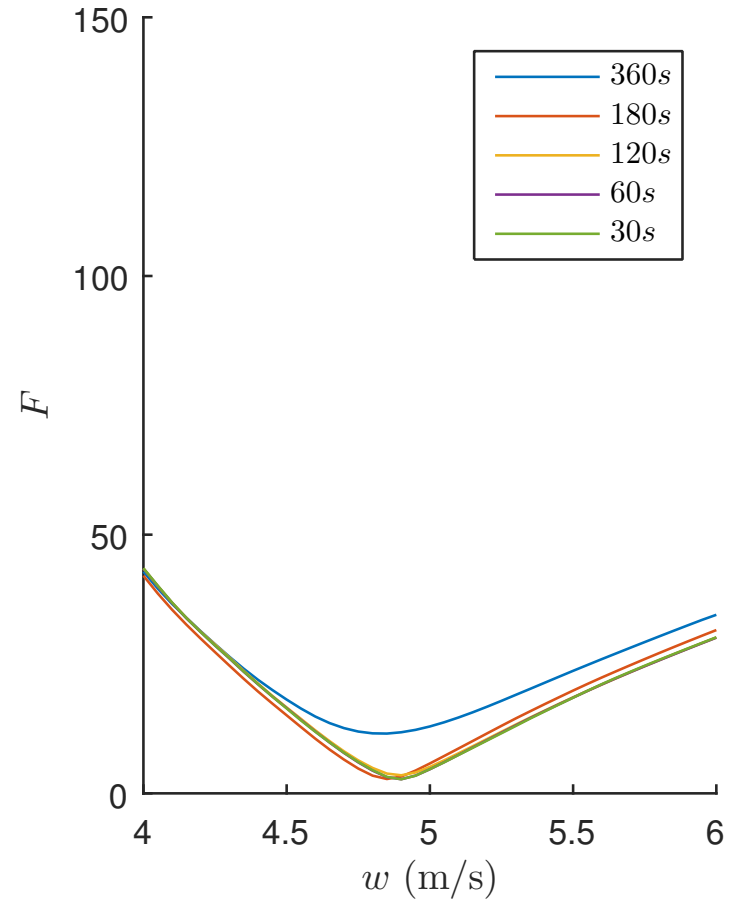

(a) $k_{x}=0.14$

Observation model 1

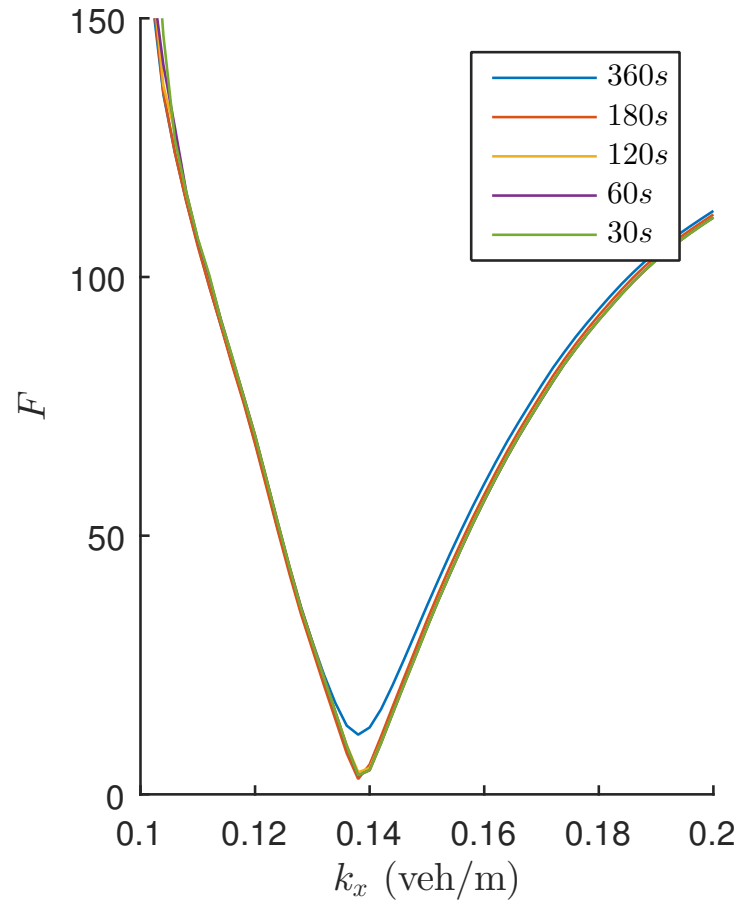

(b) $w=5$

FIGURE 6 GoF for observation model 1

- Observation model 1 : flow measurements from loop detectors with varying time resolution

- Observation model 2 : probe data with varying penetration rate

- Observation model 3 : probe data with varying frequency

According to the previous results, loop data have then been aggregated over varying time periods, from $30 s$ to $600 s$. The results are presented in Table 1 and illustrated in Figure 6.

\begin{tabular}{lcccccc}
\hline Aggregation period (s) & 30 & 60 & 120 & 180 & 360 & 600 \\
\hline$k_{x}(\mathrm{veh} / \mathrm{m})$ & 0.130 & 0.150 & 0.132 & 0.140 & 0.152 & 0.138 \\
$w(\mathrm{~m} / \mathrm{s})$ & 5.95 & 4.15 & 5.70 & 4.85 & 4.00 & 5.65 \\
$F^{*}$ & 2.700 & 2.703 & 3.483 & 2.773 & 11.53 & 885.8 \\
\hline$k_{x}^{*}(\mathrm{veh} / \mathrm{m})$ & 0.138 & 0.138 & 0.138 & 0.138 & 0.138 & 0.138 \\
$A_{k_{x}}(\mathrm{veh} / \mathrm{m})$ & 0.0023 & 0.0023 & 0.0024 & 0.0011 & 0.0026 & 0.0037 \\
$w^{*}(\mathrm{~m} / \mathrm{s})$ & 4.90 & 4.90 & 4.90 & 4.85 & 4.85 & 5.40 \\
$A_{w}(\mathrm{~m} / \mathrm{s})$ & 0.1337 & 0.1359 & 0.1517 & 0.1353 & 0.2672 & 0.4029 \\
\hline
\end{tabular}

TABLE 1 Results for observation model 1: optimal parameters, accuracy and efficiency

ime period under 180s: $F^{*}, A_{w}$ and $A_{k_{x}}$ are stable and are not impacted by the aggregation period. For aggregation time period greater than $180 \mathrm{~s}$ : the efficiency and the 
accuracy of the calibration become worse. We conclude that for practical use, loop data should be aggregated with a maximum time period of $180 \mathrm{~s}$ to ensure both efficient and accurate calibration results.

\section{Observation model 2}

Here the impact of the penetration rate of probe vehicles on the calibration results is tested. Penetration rates $\tau$ from $1 \%$ to $15 \%$ are considered. The results are presented in Table 2 and illustrated in Figure 7.

\begin{tabular}{lcccccc}
\hline$\tau(\%)$ & 1 & 3 & 5 & 7 & 10 & 15 \\
\hline$k_{x}(\mathrm{veh} / \mathrm{m})$ & 0.136 & 0.142 & 0.132 & 0.140 & 0.140 & 0.140 \\
$w(\mathrm{~m} / \mathrm{s})$ & 5.2 & 4.7 & 5.65 & 4.85 & 4.85 & 4.85 \\
$F^{*}$ & 1.969 & 2.667 & 2.168 & 2.748 & 2.773 & 2.596 \\
\hline$k_{x}^{*}(\mathrm{veh} / \mathrm{m})$ & 0.138 & 0.138 & 0.138 & 0.138 & 0.138 & 0.138 \\
$A_{k_{x}}(\mathrm{veh} / \mathrm{m})$ & 0.0013 & 0.0011 & 0.0009 & 0.0010 & 0.0011 & 0.0010 \\
$w^{*}(\mathrm{~m} / \mathrm{s})$ & 4.85 & 4.85 & 4.85 & 4.85 & 4.85 & 4.85 \\
$A_{w}(\mathrm{~m} / \mathrm{s})$ & 0.1489 & 0.1347 & 0.1224 & 0.1277 & 0.1353 & 0.1261 \\
\hline
\end{tabular}

TABLE 2 Results for observation model 2: optimal parameters, accuracy and efficiency

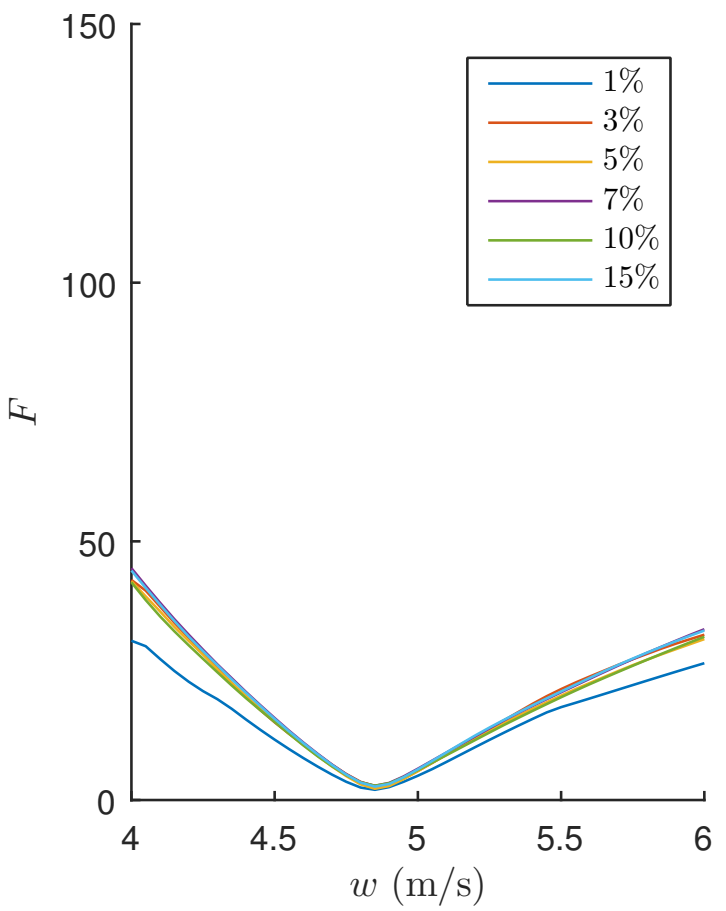

(a) $k_{x}=0.14$

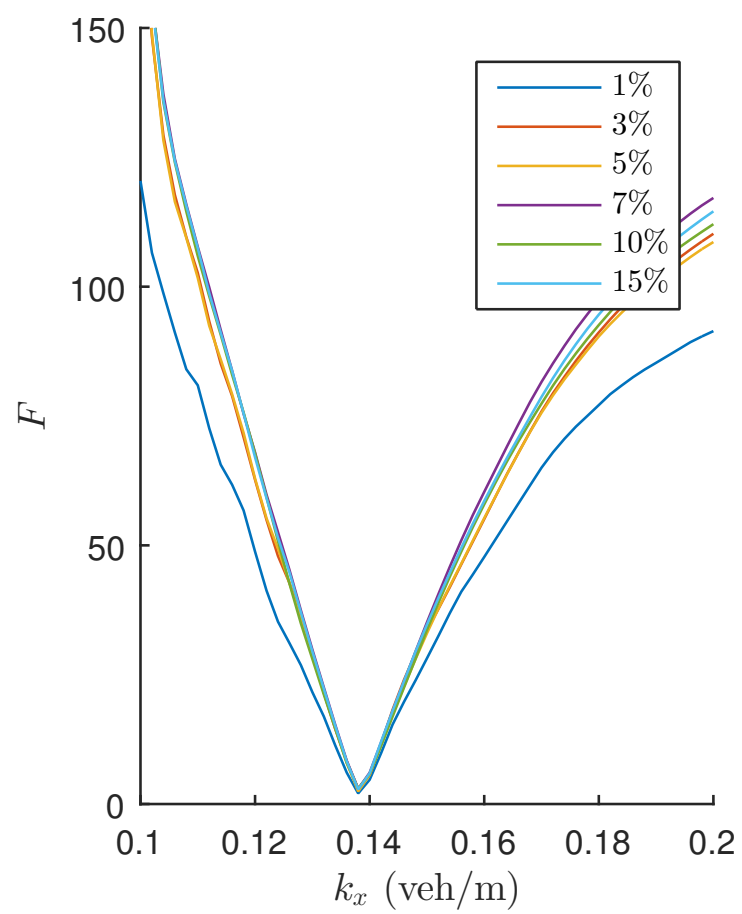

(b) $w=5$

FIGURE 7 GoF for observation model 2 
$5 \quad$ When $\tau \geq 3 \%$, the accuracy and the efficiency of the calibration process are stable. However, Figure 7 clearly shows that the accuracy of the method decreases for both parameters when $\tau=1 \%$. Nevertheless, Table 2 says that the accuracy stays in a reasonable range of value. It demonstrates that ability of this method to be developed even when the penetration rate of probe vehicles is low.

\section{Observation model 3}

Here the impact of probe data frequency is tested. Frequency from $1 / 80 s^{-1}$ to $1 / 20 s^{-1}$ are considered. The results are presented in Table 3 and illustrated in Figure 8.

\begin{tabular}{lcccc}
\hline $1 / f(\mathrm{~s})$ & 20 & 40 & 60 & 80 \\
\hline$k_{x}(\mathrm{veh} / \mathrm{m})$ & 0.132 & 0.132 & 0.140 & 0.140 \\
$w(\mathrm{~m} / \mathrm{s})$ & 5.65 & 5.65 & 4.85 & 4.85 \\
$F^{*}$ & 0.961 & 1.972 & 2.773 & 3.005 \\
\hline$k_{x^{*}}(\mathrm{veh} / \mathrm{m})$ & 0.138 & 0.138 & 0.138 & 0.138 \\
$A_{k_{x}}(\mathrm{veh} / \mathrm{m})$ & 0.0020 & 0.0012 & 0.0011 & 0.0009 \\
$w^{*}(\mathrm{~m} / \mathrm{s})$ & 4.85 & 4.85 & 4.85 & 4.85 \\
$A_{w}(\mathrm{~m} / \mathrm{s})$ & 0.1822 & 0.1385 & 0.1353 & 0.1201 \\
\hline
\end{tabular}

TABLE 3 Results for observation model 2: optimal parameters, accuracy and efficiency

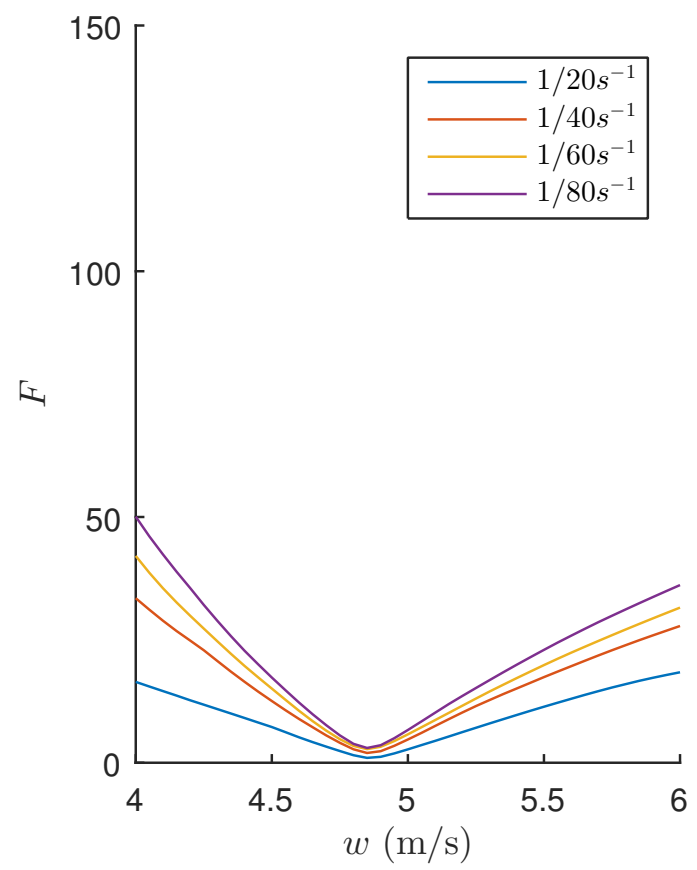

(a) $k_{x}=0.14 v e h / m$

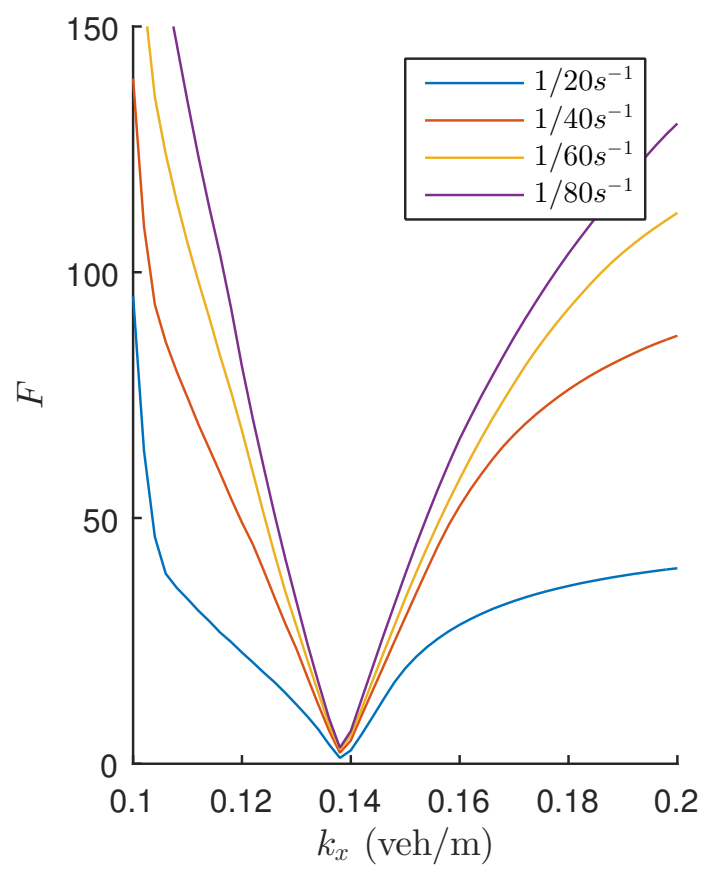

(b) $w=5 \mathrm{~m} / \mathrm{s}$

\section{FIGURE 8 GoF for observation model 3}


As expected, the efficiency of the calibration process is improved when the frequency increases. It varies within a reasonable range, from $F^{*}=3.00\left(f=1 / 80 \mathrm{~s}^{-1}\right)$ to $F^{*}=0.96$ $\left(f=1 / 20 s^{-1}\right)$.

It is clear from Figure 8 that probe data frequency has a major impact on calibration accuracy. It is confirmed in Table 3 where the best accuracy is observed for the lowest frequency $f=1 / 80 \mathrm{~s}^{-1}$. This result goes against intuition but it can be explained reasonably. When the frequency is low, probe vehicles may experience various traffic states and cross several waves between two consecutive position-time measurements. Consequently, the calibration becomes more sensitive to errors on FD parameters and thus more accurate. This result indicates that $1 / 60 \mathrm{~s}^{-1} \leq f \leq 1 / 40 \mathrm{~s}^{-1}$ is a satisfactory trade-off between accuracy and efficiency.

\section{DISCUSSION}

\section{Conclusions}

The paper presents a method for calibrating the FD parameters, assumed as being triangular, based on loop data and probe data. Loop data are considered as boundary conditions of the LWR problem. Travel distances are simulated for a range of FD parameters. Simulated travel distances are then compared to the travel distances given by probes. The optimal set of parameters is computed so that the discrepancy between simulated and collected travel distances is minimal.

Based on this methodology, the calibration procedure has been tested on an error free dataset and it has demonstrated the accuracy of the calibration procedure. Then it has been tested for different observation model, to estimate the impact of data resolution on calibration results, which have been analyzed regarding two indicators : accuracy and efficiency. The results shows that common loop data are proper to apply the methodology. It shows that the performance of the method is satisfactory for a penetration rate of probe vehicle greater than $3 \%$, which is an encouraging but also must be confirmed with further experiments based on real dataset. It also shows that, counterintuitively, high-frequency probe data do not necessary provide more accurate results.

\section{Further Research}

The proposed methodology has been applied and validated based on simulated noised data with various resolution. However, further research is still needed to confirm its practical use when applied to real data.

- The proposed methodology estimates traffic states between loop detectors based on the method of characteristics. Other technics have been proposed in the literature, notably based on variational principles, and can be explored and compared in terms of computational efficiency.

- In the paper, the GoF is defined so that errors from low speed probes receive heavier weights than errors from probes driving close to the free-flow speed. Further analysis should be conducted to validate the weight definition when applied to real data.

- Here, data from loops have been noised considering an unbiased random undetected / doubledetected vehicles but vehicles keep the same ordering between the loops (First In First Out, FIFO). This assumption may not be met on multilane sections with multi pipe regimes and further research is needed to test the robustness of the method when applied to non-FIFO traffic flow.

- It is assumed that the road section does not present any entry or exit between loop detectors. Here the methodology can be adapted to estimate the net additional flow that enters/exits the section between loops based on (i) the data from loops and probes and (ii) assuming a given (default or pre-calibrated) fundamental diagram. Such a methodology can be useful to estimate the exact entry 
(exit) flow from on-ramp (off-ramp) when congestion occurs between loops.

Finally, the proposed methodology can be used for off-line application. But the computational efficiency of the methodology is promising and it can also be the basis for developing an algorithm that calibrates automatically FD parameters online. 


\section{REFERENCES}

[1] J. Castillo, F. Benítez, On the functional form of the speed-density relationship-ii: Empirical investigation, Transportation Research Part B: Methodological 29 (5) (1995) 391 - 406. doi:http://dx.doi.org/10.1016/0191-2615 (95)00009-3. URL http://www.sciencedirect.com/science/article/pii/0191261595000093

[2] M. Van Aerde, H. Rakha, Multivariate calibration of single regime speed-flow-density relationships, in: Proceedings of the 6th 1995 Vehicle Navigation and Information Systems Conference, 1995, pp. 334-341.

[3] H. Rakha, B. Crowther, Comparison of greenshields, pipes, and van aerde car-following and traffic stream models, Transportation Research Record: Journal of the Transportation Research Board (1802) (2002) 248-262.

[4] L. Leclercq, Calibration of flow-density relationships on urban streets, Transportation Research Record: Journal of the Transportation Research Board (1934) (2005) 226-234.

[5] N. Chiabaut, C. Buisson, L. Leclercq, Fundamental diagram estimation through passing rate measurements in congestion, Intelligent Transportation Systems, IEEE Transactions 10 (2).

[6] M. J. Cassidy, R. L. Bertini, Some traffic features at freeway bottlenecks, Transportation Research Part B: Methodological 33 (1) (1999) 25 - 42. doi:http://dx.doi.org/ $10.1016 /$ S0191-2615 (98)00023-X. URL http://www.sciencedirect.com/science/article/pii/S019126159800023X

[7] N. Chiabaut, L. Leclercq, Wave velocity estimation through automatic analysis of cumulative vehicle count curves, Transportation Research Record: Journal of the Transportation Research Board 2249.

[8] http://ngsim-community.org.

[9] S. Ossen, S. Hoogendoorn, B. Gorte, Interdriver differences in car-following: a vehicle trajectory-based study, Transportation Research Record: Journal of the Transportation Research Board (1965) (2006) 121-129.

[10] S. Ossen, S. Hoogendoorn, Validity of trajectory-based calibration approach of car-following models in presence of measurement errors, Transportation Research Record: Journal of the Transportation Research Board (2088) (2008) 117-125.

[11] A. Duret, C. Buisson, N. Chiabaut, Estimating individual speed-spacing relationship and assessing ability of newell's car-following model to reproduce trajectories, Transportation Research Record: Journal of the Transportation Research Board (2088) (2008) 188-197.

[12] M. Treiber, A. Kesting, Microscopic calibration and validation of car-following models - a systematic approach, Procedia - Social and Behavioral Sciences 80 (0) (2013) 922 - 939, 20th International Symposium on Transportation and Traffic Theory (ISTTT 2013). doi: http://dx.doi.org/10.1016/j.sbspro.2013.05.050. URL http://www.sciencedirect.com/science/article/pii/S1877042813010197 
[13] N. Chiabaut, L. Leclercq, C. Buisson, From heterogeneous drivers to macroscopic patterns in congestion, Transportation Research Part B: Methodological 44 (2) (2010) 299 - 308. doi:http://dx.doi.org/10.1016/j.trb.2009.07.009. URL http://www.sciencedirect.com/science/article/pii/S0191261509000976

[14] J. C. Herrera, A. M. Bayen, Traffic flow reconstruction using mobile sensors and loop detector data, University of California Transportation Center.

[15] A. Bhaskar, E. Chung, A. Dumont, Fusing loop detector and probe vehicle data to estimate travel time statistics on signalized urban networks, Computer-Aided Civil and Infrastructure Engineering 26 (6) (2011) 433-450.

[16] N.-E. El Faouzi, H. Leung, A. Kurian, Data fusion in intelligent transportation systems: Progress and challenges-a survey, Information Fusion 12 (1) (2011) 4-10.

[17] L. Klein, L. Mihaylova, N.-E. El Faouzi, Handbook on Soft Computing for Video Surveillance, Chapman and Hall/CRC Cryptography and Network Security Series, Taylor and Francis, 2012, Ch. Sensor and Data Fusion: Taxomany, Challenges and Applications.

[18] M. J. Lighthill, G. B. Whitham, On kinematic waves. ii. a theory of traffic flow on long crowded roads, Proceedings of the Royal Society of London A: Mathematical, Physical and Engineering Sciences 229 (1178) (1955) 317-345. doi:10.1098/rspa.1955.0089.

[19] P. I. Richards, Shock waves on the highway, Operations research 4 (1) (1956) 42-51.

[20] C. F. Daganzo, A finite difference approximation of the kinematic wave model of traffic flow, Transportation Research Part B: Methodological 29 (4) (1995) 261 - 276. doi:http: / / dx.doi.org/10.1016/0191-2615(95)00004-W. URL http://www.sciencedirect.com/science/article/pii/019126159500004W

[21] L. Leclercq, C. Becarie, Meso lighthill-whitham and richards model designed for network applications, Tech. rep. (2012).

[22] J. A. Laval, L. Leclercq, The hamilton-jacobi partial differential equation and the three representations of traffic flow, Transportation Research Part B: Methodological 52 (2013) 17-30.

[23] Y. Yuan, A. Duret, H. Van Lint, Mesoscopic traffic state estimation based on a variational formulation of the lwr model in lagrangian-space coordinates and kalman filter, in: Transportation Procedia, Elsevier, 2015.

[24] A. Duret, C. Buisson, N. Chiabaut, Estimating individual speed-spacing relationship and assessing ability of newell's car-following model to reproduce trajectories, Transportation Research Record: Journal of the Transportation Research Board (2088) (2008) 188-197 03611981.

[25] A. Duret, Measurement of variability involved in the car-following rules, in: European Conference on Transportation Research Institutes - Young Researcher Seminar, 2009. 